\title{
MiR-29a and miR-652 Attenuate Liver Fibrosis by Inhibiting the Differentiation of CD4+ T Cells
}

\author{
Ji Xuan ${ }^{1,2 \dagger}$, Shu-lin Guo ${ }^{3 \dagger}$, Ang Huang ${ }^{4 \dagger}$, Hua-bing Xu' ${ }^{1}$, Mei Shao ${ }^{1}$, Ya Yang${ }^{1}$, and Wei Wen ${ }^{*}$ \\ ${ }^{1}$ Bayi Hospital Affiliated Nanjing University of Chinese Medicine, Nanjing, 210002, China, ${ }^{2}$ Nanjing \\ University of Chinese Medicine, Nanjing, 210046, China, ${ }^{3} 107$ Hospital of PLA, Yantai, 264000, China, \\ ${ }^{4}$ Center of Non-infectious Liver Diseases, Beijing 302 Hospital, 100000, China
}

\begin{abstract}
Background: Liver fibrosis is the response of liver diseases that puzzles patients. MiRNAs were involved in the regulating processes of liver fibrosis. This study aims to investigate the effects of ARRB1 mediated by miR-29a and miR-652 on liver fibrosis and its possible mechanism. Methods: Liver fibrosis of mice was induced by intraperitoneal injection of $\mathrm{CCl}_{4}$. Liver function was observed by the levels of alanine transaminase (ALT) and aspartate transaminase (AST). Flow cytometry was used to detect the percent of T helper17 (Th17). ELISA (Enzyme linked immunoassay) was used to detect the levels of Interleukin-17 (IL-17) and Interleukin-22 (IL-22). Real-time PCR was used to detect the expression of IL-17A, IL-22, miR-29a, miR-652 and $\beta$-Arrestin 1 Gene (ARRB1). Western blot was used to detect the protein expression of ARRB1. Results: $\mathrm{CCl}_{4}$ supplementation significantly increased the level of ALT and AST, the percent of Th17, the level of IL-17A, IL-22, miR-29a and miR-652, but decreased ARRB1. Overexpression of miR-29a/miR-652 prominently decreased Th17, IL-17A, IL-22 and ARRB1 in the normal CD4+ T cells. Both miR-29a and miR-652 targeted ARRB1 to regulate its expression. The effects of miR-29a/miR-652 overexpression on CD4+ $T$ cells were reversed by ARRB1 overexpression. In vivo experiments demonstrated the protective role of $\mathrm{miR}-29 \mathrm{a} / \mathrm{miR}-652$ overexpression on liver fibrosis. Conclusion: ARRB1 mediated by miR-29a and miR-652 probably involved in the CD4+ $T$ cells differentiation in patients with liver fibrosis, and functioned as a biomarker of fibrosis liver.
\end{abstract}

Key words: liver fibrosis, miR-29a, miR-652, ARRB1, CD4+ T cells

\section{Introduction}

Liver fibrosis is always considered as the response of various types of liver injuries, characterized by excessive production of extracellular matrix (ECM), which leads to the destruction of liver parenchym (Hernandez-Gea and Friedman, 2011). Patients suffering from liver fibrosis are easy to developing into liver cirrhosis, which was associated with severe mortality (Pellicoro et al., 2014; Kochanek et al., 2016). Although much improvement has been made on the patients with liver fibrosis based on the prevalent therapeutic methods, such as radiotherapy, chemotherapy and surgery, the prognosis remains poor (Justinger et al., 2015; Li et al., 2012a; Moretto et al., 2012). However, to fully understand the pathogenesis of fibrosis is necessary for its further treatment.

\footnotetext{
These authors contributed equally to this work.

*To whom correspondence should be addressed: Wei Wen, Bayi Hospital Affiliated Nanjing University of Chinese Medicine, No. 34 Yang Gong Jing 34 District, Nanjing, 210002, China.

Tel: +86-025-85816332

E-mail: wenweiabc@yeah.net
}

CD4+ T cells were a set of cells that involved in the host immune system through liberating the cytokines such as $\mathrm{T}$ helper17 (Th17) (Schenten et al., 2014). Recently study has proved that the percent of $\mathrm{CD} 4+\mathrm{T}$ cells were affecting the liver inflammation and fibrosis (Zhang et al., 2013). Interleukin-17 (IL-17) is a pro-inflammatory cell that plays vital role on host defenses and several autoimmune disorders through releasing of cytokines such as IL-17A and Interleukin-22 (IL-22), and both IL-17A and IL-22 functioned to control of extracellular bacterial infection (Shigematsu et al., 2013). Studies have demonstrated that the level of Th17 cells were significantly increased in patients with liver diseases, additionally, the increasingly level of Th17 cells also promoted the progression of liver fibrosis (Li et al., 2012b). While the levels of IL-17A and IL-22 in the liver tissues were always considered as the main factors to measure liver function. For example, studies have reported that the imbalance of Th17/Treg was the main marker of liver fibrosis (Sun et al., 2014; Gu et al., 2016). Another study have identified that the frequency of IL-17A and IL-22 could altered liver fibrogensis via hepatic stellate cell activation and inflammatory cytokines 
secretion (Gu et al., 2016).

MicroRNA (miRNA) was a class of endogenous noncoding RNA with the length of $\sim 22 \mathrm{nt}$, and functioned to control the target genes expression by binging with the mRNAs 3'UTR (Vishnoi and Rani, 2017). Recent studies have demonstrated that a single miRNA could target one or more mRNA to regulate their expression, while an mRNA also could be targeted by several miRNAs in the biological processes(Guo et al., 2014; Bruce and Liu, 2014). However, the complex networks composed of the whole regulation system of the life. Much studies have reported that miRNAs expression profiling were involves in a variety of human diseases, they can act as pathogenic genes or resistant genes (Yan et al., 2014; Van De Bunt et al., 2013; Xu et al., 2015; Wu et al., 2016). In human liver fibrosis, a number of miRNAs have been well documented, such as miR-20a, miR-21, miR-122 (Roderburg and Luedde, 2014). MiR-29A was an important biomarker in patients with liver fibrosis, the expression of which was significantly decreased in the serum of fibrosis patients comparing with the healthy person(Roderburg et al., 2011). Another significant biomarker of liver fibrosis miR-652 has been verified to play an important role in the fibrogenesis and inflammatory processes in liver cirrhosis(Roderburg et al., 2012). Nevertheless, whether miR-29a and miR-652 could modulate the progression of liver fibrosis and the underlying mechanism were still unclear. This study, we aim to explore the potential role of miR-29a and miR-652 on liver fibrosis, which would provide great help for the clinical therapy.

\section{Materials and Methods}

\section{Animals}

A total of 16 healthy male C57BL/6 mice aged 4-6 weeks old were purchased from Shanghai BK Experimental Animal Center. All mice were raised under same environment with pathogen-free conditions, adequate food and water, as well as cosy temperature and humidity. The experiment was conformed to the Guide for the Care and Use of Laboratory Animals and were permitted by the Research Ethics Committee.

\section{Establishment of hepatic fibrosis mice model}

The mice were randomly divided into 2 groups, including $\mathrm{CCl}_{4}$ induced model group and normal control group. The model mice was intraperitoneally injected with $1 \mathrm{ml} / \mathrm{kg}$ of $10 \% \mathrm{CCl}_{4}$, which dissolved in olive oil. The injection was performed 3 times a week up to 3 weeks. The control mice were injected with a equal amount of olive oil. After three weeks, all mice were sacrificed by euthanasia method, the samples were collected for the following experiments.

\section{Detection of ALT and AST}

Alanine transaminase (ALT) and aspartate transaminase (AST) kit were purchased from Guidechem biotechnology (China). The levels of ALT and AST were detected according to the manufacture's instruction.

\section{Cell isolation and culture}

In order to obtain CD4+ T cells, liver tissues collected from the mice were grinded and centrifuged. Then CD4+ T Cell Isolation Kit (Miltenyi Biotec, Auburn, CA, USA) was used to isolate the single-cell of CD4+ T by depletion of non-CD4+ T cells according to the manufacture's instruction. The cells were maintained with RPMI-1640 medium supplemented with $10 \%$ FBS at $37^{\circ} \mathrm{C}$ with $5 \% \mathrm{CO} 2$ in a humid environment.

\section{CD4+ T cell activation and differentiation}

CD4+ T cells isolated from liver tissues of normal mice were activated by $5 \mathrm{mg} / \mathrm{ml}$ anti-CD3 (Abcam) and $2 \mathrm{mg} / \mathrm{ml}$ soluble antiCD28 (Abcam) and then induced to differentiate into Th17 by the medium containing of $2.5 \mathrm{ng} / \mathrm{ml}$ rTGF- $\beta 1,30 \mathrm{ng} / \mathrm{ml} \mathrm{rIL-6,} 10$ $\mathrm{mg} / \mathrm{ml}$ anti-IFN- $\gamma$, and $10 \mathrm{mg} / \mathrm{ml}$ anti-IL-4.

\section{Flow cytometry assay}

For detecting the level of Th17, a FACS Calibur flow cytometer (BD Bioscience) was used. Briefly, cells were incubated with phorbol myristate acetate (PMA) for $4 \mathrm{~h}$ and then fixed in $2 \%$ paraformaldehyde, then FITC-labeled anti-mouse CD4 was used to stain the cells at $4{ }^{\circ} \mathrm{C}$ for $30 \mathrm{~min}$. Next, cells were fixed, permeabilized and stimulated by PE-labeled anti-IL-17A in the dark for another $20 \mathrm{~min}$. All regents in this study were purchased from Nanjing Shoude biotech CO., LTD (Nanjing, China).

\section{Enzyme-linked immunoassay (ELISA)}

After CD4+ T cells were activated with or without transfected, cells culture supernatant were collected for detecting the level of IL-17 and IL-22. ELISA kits of IL-17 and IL-22 (Nanjing Shoude biotech CO., LTD.) were used according to the manufacture's instruction.

\section{Real-time PCR}

Total RNA was isolated from the cells or tissues with TRIzol regent (Invitrogen, USA) and an Nanodrop 2000 spectrophotometer (USA) was used for RNA quantification. Then $1 \mu \mathrm{L}$ of RNA was taken out for the reverse transcription by using of Reverse Transcription Kit (Qiangen, Germany). Real-time PCR was performed on an ABI 7900HT Fast Real-time PCR (Applied Biosystems USA) based on the RT-PCR Kit (Takara) according to the manufacture's instruction. The primers used in this study were synthesized from Yingjun Technology CO., LTD (Shanghai, 
China). U6 served as internal control. The primers of miR-29a and miR-652 were as following:

miR-652:

forward: 5'-ACACTCCAGCTGGGCAACCCTAGGAGAGGGTGC-3' reverse: 5'-GTGTCGTGGAGTCGGCAATTC-3'

miR-29a:

forward: 5'-TGCGCTAGCACCATCTGAAAT-3'

reverse: 5'-CAGTGCAGGGTCCGAGGT-3'

\section{Cell transfection}

Cells were cultured in the 96-well plate for 1 day to ensure a suitable cell confluent, the pre-NC or miR-29a mimic or miR-652 mimic or miR-29a inhibitor or miR-652 inhibitor were transfected into cells with Lipofectamine 2000 reagent, according to the manufacturer's protocol. The mimics, inhibitors and NCs were synthesized in Shanghai Yingjun Co., Ltd. (China). The ARRB1 overexpression plasmid was constructed to transfect into cells, the empty plasmid was recognized as control.

\section{Western blot}

Cells or tissues were washed with ice-cold phosphate-buffered saline (PBS) and then lysed with RIPA lysis buffer, and centrifuged to collect the protein extracts. The protein concentration was detected by Bradford method. SDS-PAGE (sodium dodecyl sulfate polyacrylamide gel electrophoresis) was used to separate the protein extracts. Then an equal amount of protein was transferred to polyvinylidene fluoride (PVDF) membrane, and the membrane was incubated with anti-ARRB1 ( $\beta$-Arrestin 1 Gene) (1:5000, Sigma) at $4{ }^{\circ} \mathrm{C}$ for one night. Next, the blots were incubated with secondary antibodies for another $2 \mathrm{~h}$ at room temperature. GAPDH acted as internal control. Immunoblot was visualized by using ECL detection kit (Invitrogen) according to the manufacturer's instruction. All the experiments were performed three individual times.

\section{Luciferase reporter assay}

The sequence of ARRBI was chose from an online Genbank. The ARRBI 3'UTR was synthesized and cloned into the specific vector to construct the luciferase reporter plasmid of Luc-RBPJ-3'UTR-WT and Luc-RBP-J-3'UTR-Mut. Lipofectamine2000 transfection reagent was used for the transfection, according to the manufacture's transfection. The relative luciferase activity was measured by Dual-Luciferase Reporter Assay.

\section{Induction of liver fibrosis in vivo}

A total of 24 healthy C57BL/6 mice with 4-6 weeks old were randomly divided into 3 groups, including Ad-control group, AdmiR-29a and Ad-miR-652 group. The Adenovirus vectors of miR-29a and miR-652 were constructed in ZhongHongBoYuan Co., Ltd. (China). The mice were tail injected with miR-29a or miR-652 overexpression plasmid twice a week up to 10 weeks.
The injection dose of Ad-miR-29a and Ad-miR-652 was $100 \mu \mathrm{L}$ in each time. Then the CD4+ T cells was isolated and in vivo to detect the level of ALT and AST, the ratio of Th17, and the expression level of ARRB1.

\section{Statistical ananlysis}

ANOVA was used for the comparison between the groups, while Student's $t$ test were used for the comparison of paired data. Data were expressed as means $\pm \mathrm{SD}$. All results represented at least three independent experiments. ${ }^{*} \mathrm{P}<0.05$ was considered as statistically significant.

\section{Results}

\section{$\mathrm{CCl}_{4}$ induced changes of liver function in mice}

To examine the effect of $\mathrm{CCl}_{4}$ on liver function, the serum of mice in the two groups were collected. According to the Fig. 1, we found that $\mathrm{CCl}_{4}$ administration significantly increased the levels of ATL (Fig. 1A) and AST (Fig. 1B) compared with control. Then the CD4+ T cells of liver tissues were isolated from the control mice and $\mathrm{CCl}_{4}$ induced hepatic fibrosis mice. We found that the Th17 secreted from CD4+ $\mathrm{T}$ cells was significantly higher in $\mathrm{CCl}_{4}$ induced hepatic fibrosis mice rather than in the normal mice (Fig. 1C). Then the ELISA and real-time PCR were used to detect the level and mRNA expression of IL-17A and IL-22, the results revealed that $\mathrm{CCl}_{4}$ stimulation significantly increased the level and the expression of IL-17A and IL-22 (Fig. 1D and 1E).

\section{Low-expressed miR-29a and miR-652 and up- regulated $A R R B 1$ in $C_{C l}$ induced hepatic fibrosis model}

To examine the expression pattern of miR-29a, miR-652 and ARRB1, the RNA and protein were extracted from liver tissues of both model and control mice. Real-time PCR demonstrated that the expression level of miR-29a and miR-652 in model group was significantly decreased (Fig. 2A and 2B), while the ARRB1 mRNA level was significantly increased (Fig. 2C). Western blotting presented that the protein expression of ARRB1 was dramatically increased in model group compared with control (Fig. 2D).

\section{Overexpression of miR-29a or miR-652 decreased IL-17A, IL-22 and ARRBI}

CD4+ T cells of isolated from healthy mice were transfected with miR-29a mimic or miR-652 mimic, after that, cells were activated cultured and induced to secrete Th17 cells. As was shown in Fig. 3, both overexpression of miR-29a and miR-652 significantly decreased the percent of Th17 
A

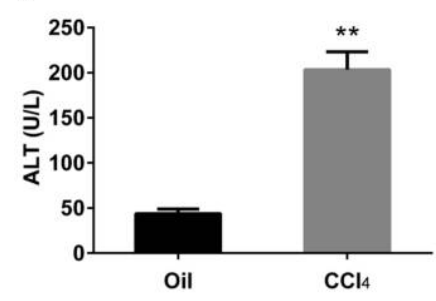

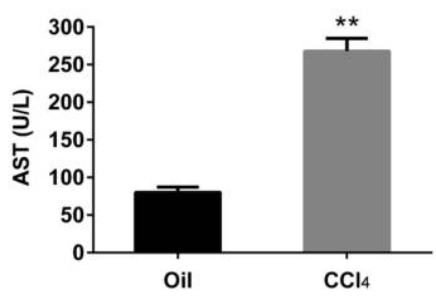

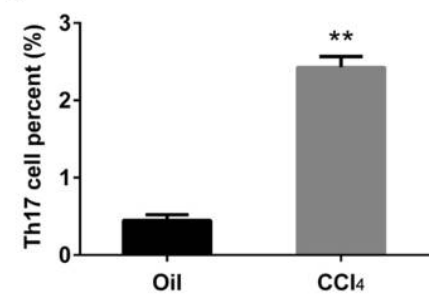

D

E
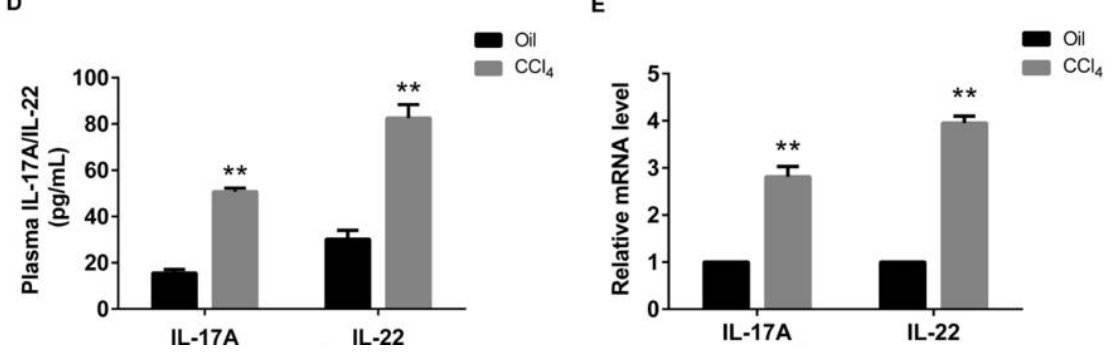

Fig. 1. Elevated levels of ALT, AST, Th17 and its cytokines in mice with liver fibrosis. A and B: the levels of ALT and AST were significantly increased in $\mathrm{CCl}_{4}$ induced fibrosis liver than the control. $\mathrm{C}$ : $\mathrm{CCl}_{4}$ supplementation increased the percent of Th17 cells in CD4+ T cells. D and E: ELISA and realtime PCR were used to detect the levels and expressions of IL-17 and IL-22 in fibrosis liver and normal control. **P $<0.01$ vs control (Oil)

A

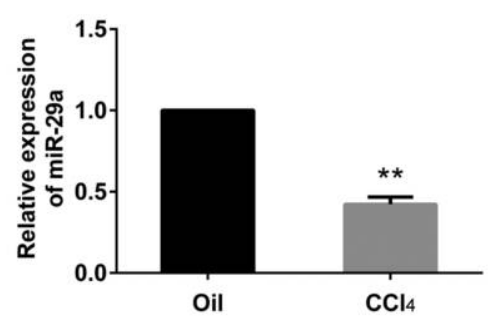

C

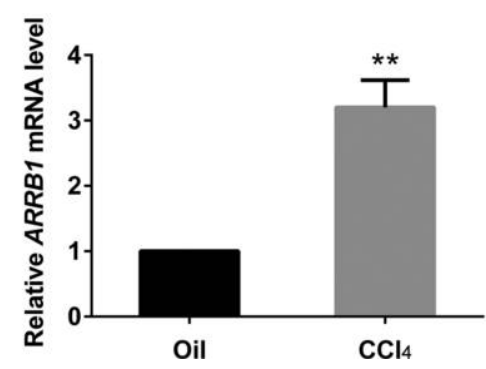

B

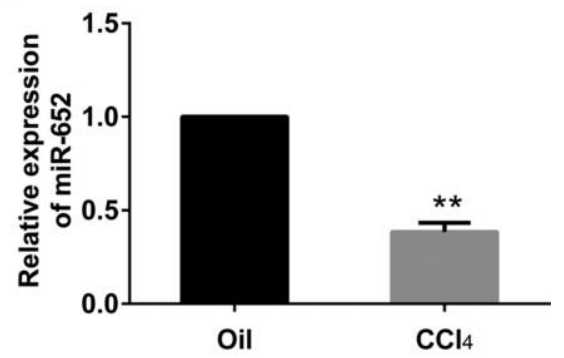

D

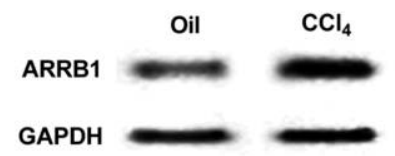

Fig. 2. The expressions of miR-29a, miR-652 and ARRB1 in $\mathrm{CCl}_{4}$-induced liver fibrosis of mice. $\mathrm{A}$ and B: real-time PCR reflected the decreased expression of miR-29a and miR-652 in the fibrosis mice. C and D: the expression pattern of ARRB1 was presented by real-time PCR and western blot. $* * \mathrm{P}<0.01$ vs control (Oil), GAPDH served as internal control.

cells (Fig. 3A), the levels of IL-17A and IL-22 (Fig. 3B), the mRNA expressions of IL-17A, IL-22 and ARBB1 (Fig. $3 \mathrm{C}$ and $3 \mathrm{D})$, as well as the protein expression level of ARRB1 (Fig. 3E).

\section{Both miR-29a and miR-652 targeted ARBB1 to regulate its expression}

To verify the relationship between ARRB1 and miR-29a or miR-652, an online database of target protector was used, 


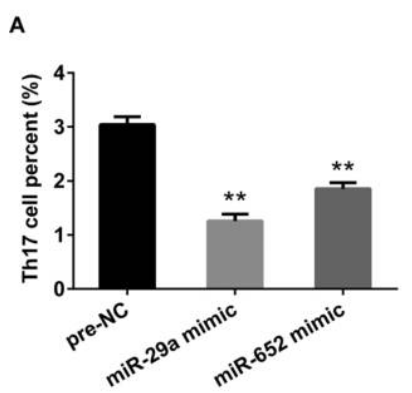

D
B

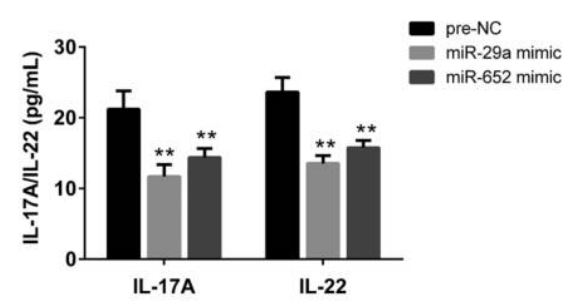

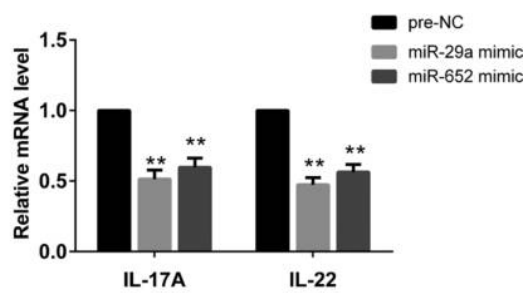

E
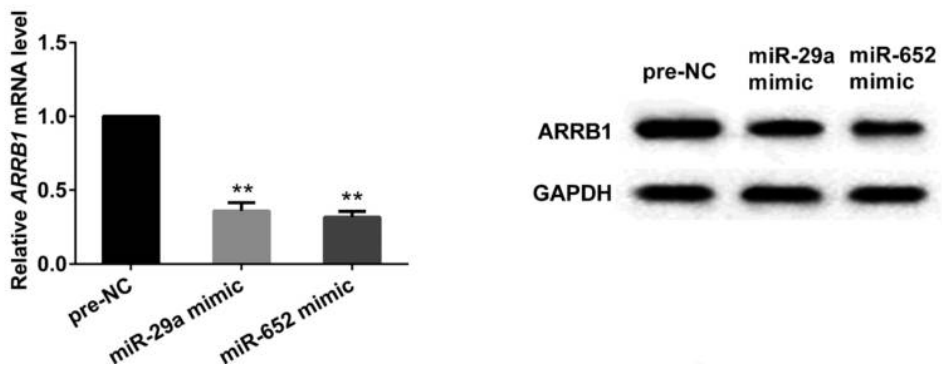

Fig. 3. Up-regulated miR-29a/miR-652 ameliorated liver fibrosis. CD4+ T cells transfected with miR-29a mimic or miR-652 significantly decreased the percent of Th17 cells (A), the levels of IL-17A and IL-22 (B), the mRNA expression of IL-17A and IL-22 (C), the mRNA and protein expression of ARRB1 (D and E). **P<0.01 vs pre-NC, GAPDH served as internal control.

A

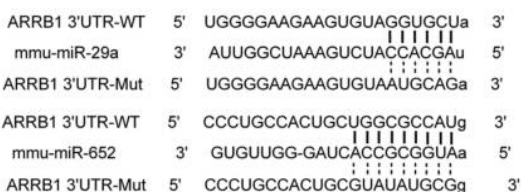

D

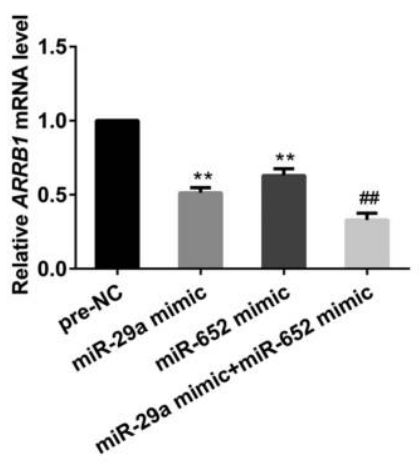

B

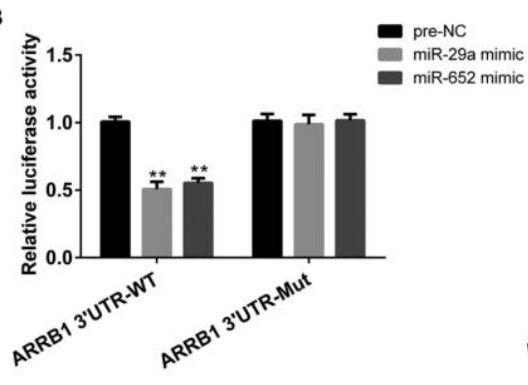

$\mathbf{F}$

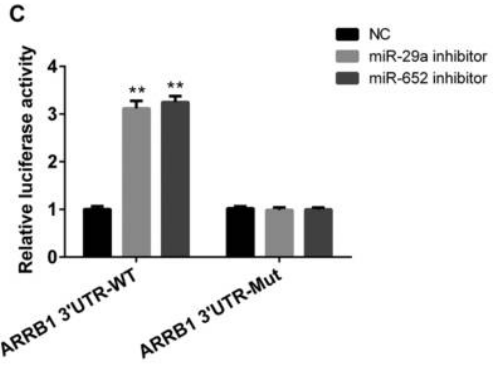

E

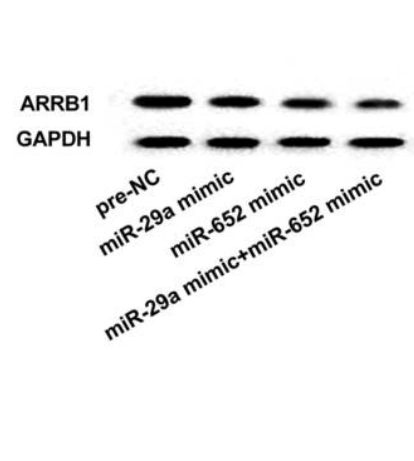

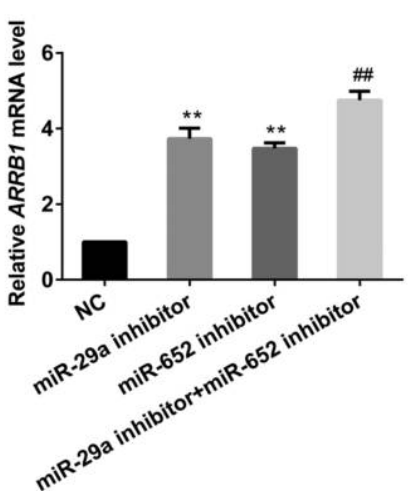

G

Fig. 4. Both miR-29a and miR-652 targeted ARRB1. A: online database predicted that both miR-29a and miR-652 binding with 3'UTR of ARRB1. Luciferase reporter assay verified the targeted relationships of miR-29a and ARRB1 (B), as well as miR-652 and ABBR1 (C). MiR-29a overexpression or miR-652 overexpression significantly decreased the mRNA and protein expression of ARRB1, and the cooperation of MiR-29a overexpression and miR-652 overexpression strengthened the effects (D and E). MiRNA-29a knockdown and miR-652 knockdown significantly increased the mRNA and protein expression of ARRB1, while the cooperation of MiR-29a and miR-652 strengthened the effects ( $\mathrm{F}$ and $\mathrm{G})$. ${ }^{* *} \mathrm{P}<0.01$ vs pre-NC (NC); ${ }^{\#} \mathrm{P}<0.01$ vs miR-29a/miR-652 mimic or miR-29a/miR-652 inhibitor, GAPDH served as internal control. 
A

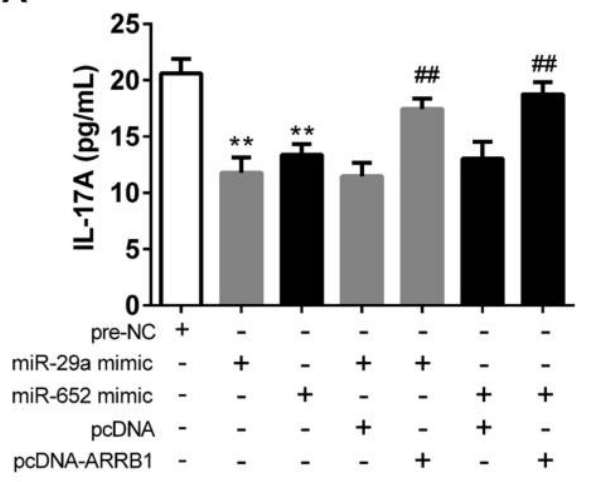

C

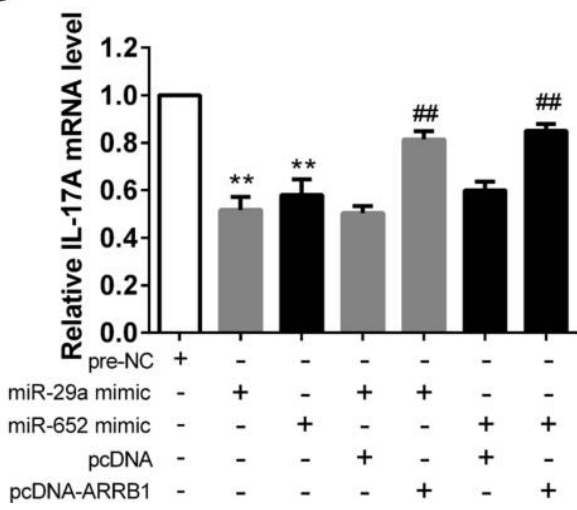

B

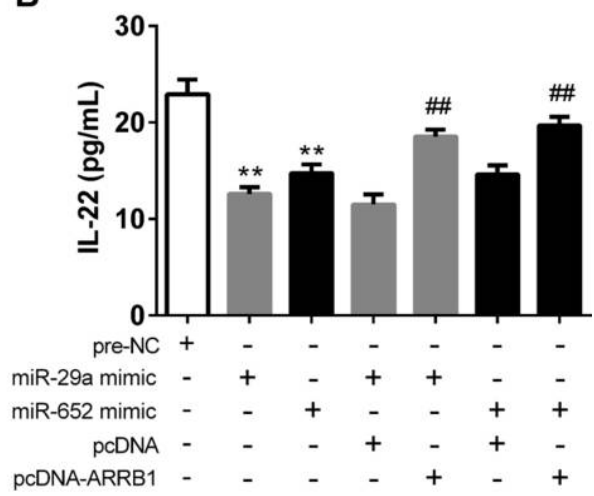

D

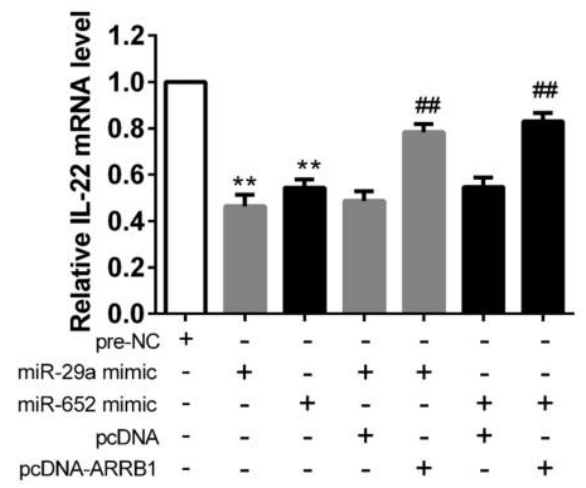

Fig. 5. Effects of ARRB1 overexpression on normal CD4+ T cells with administration of miR-29a mimic or miR-652 mimic. A and B: The levels of IL-17A and IL-22 were detected by ELISA. C and D: the mRNA expressions of IL-17A and IL-22 were examined by real-time PCR. $* * P<0.01$ vs pre-NC \#\# $\mathrm{P}<0.01$ vs miR-29a/miR-652 mimic + pcDNA.

as presented in Fig. 4A, both miR-29a and miR-652 could target the ARRB1 3'UTR-WT. Relative reporter assay found that both miR-29a mimic and miR-652 mimic could significantly decreased the luciferase activity of ARRB1 3'UTR-WT (Fig. 4B), while miR-29a inhibitor or miR-652 inhibitor could prominently increased the luciferase activity of ARRB1 3'UTR-WT (Fig. 4C).

CD4+ T cells isolated from healthy mice were transfected with miR-29a mimic or miR-652 mimic or miR-29a mimic + miR-652 mimic, which could significantly decreased the mRNA and protein expression of ARRB1 (Fig. 4D and 4E). While CD4+ T cells transfected with miR-29a inhibitor or miR-652 inhibitor or miR-29a inhibitor+miR-652 inhibitor could remarkably increased the mRNA and protein expression of ARRB1 (Fig. 4F and 4G).

\section{Interaction effects of miR-29a/miR-652 and ARRB1 on liver function}

We then examined whether miR-29a/miR-652 mediates ARBB1 to be involved in the liver function in CD4+ T cells. Cells transfected with miR-29a mimic/miR-652 mimic significantly decreased the level and mRNA expression of IL-17A and IL-22, whereas, they were dramatically increased after the supplementation of pcDNA-ARRB1 (Fig. 5).

\section{In vivo to detect the effects of miRNAs overexpression on liver function}

Healthy male C57BL/6 were intraperitoneal injected of $\mathrm{CCl}_{4}$ to induce hepatic fibrosis mice. Then the mice were randomly divided into 3 groups including control group and model groups, while the model groups including the AdmiR-29a group and Ad-miR-652 group by tail injection of adenovirus expression vector of miR-29a or miR-652. Results revealed that in CD4+ T cells, both overexpression of miR-29a and miR-652 could significantly decrease the level of ALT and AST, the percent of Th17 cells, as well as the miRNA and protein expression of ARRB1 (Fig. 6). 
A

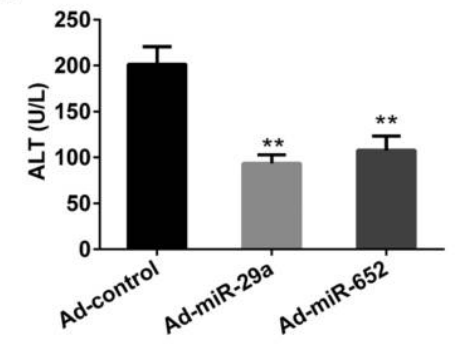

B

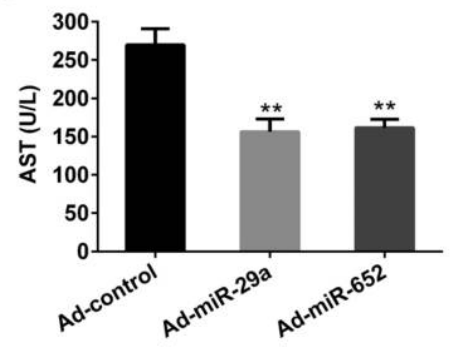

C

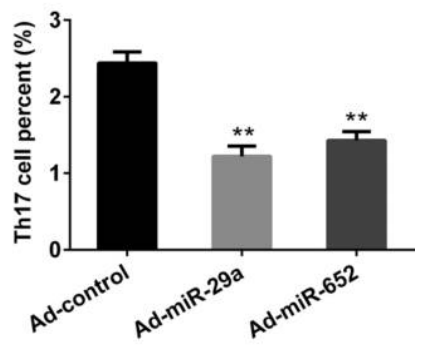

D

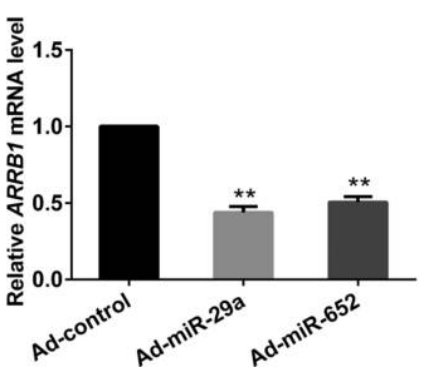

E

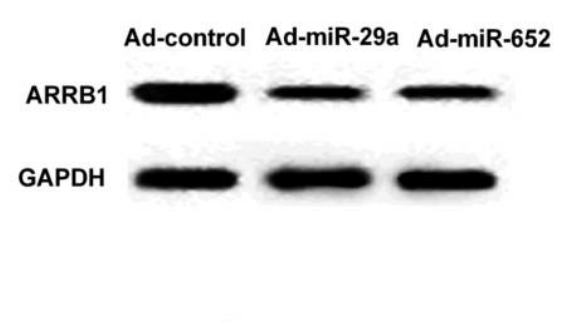

Fig. 6. In vivo to verify the effects of miR-29a overexpression/miR-652 overexpression. miR-29a overexpression/miR-652 overexpression significantly decreased the level of ALT and AST (A and B), the percent of Th17 (C), the mRNA and protein expression of ARRB1 (D and E) . **P<0.01 vs Adcontrol, GAPDH served as internal control.

\section{Discussion}

$\mathrm{CCl}_{4}$ supplementation to mice always induced several liver symptoms that similar with the human liver fibrosis, thus, $\mathrm{CCl}_{4}$ stimulation in mice has been employed as a model of liver fibrosis (Park et al., 2015). From this study, we found that $\mathrm{CCl}_{4}$ stimulation significantly increased the percent of Th17, the level of IL-17A, IL-12, as well as the expression of ALT and AST, previous study has proven that the fibrosis could induce high level of IL-17A and IL-12, in addition, the expressions of ALT and AST were the main factors that reflected the liver function, while the abnormal increase of ALT and AST means the dysfunction of liver. All those evidences indicated the successful model we have established.

$\beta$-arrestins belongs to the family of arrestin, the classical functions of $\beta$-arrestins are involved in the desensitization and recycling of $\mathrm{G}$ protein-coupled receptors (Smith and Rajagopal, 2016), while increasingly researchers suggested that $\beta$-arrestins also can to modulate intracellular functions, for example, Sun et al found that $\beta$-arrestin2 was involved in the proliferation of hepatic stellate cells via ERK pathway (Sun et al., 2013), while Li et al suggested that $\beta$ arrestin1 attenuate arthritis through impairing the differentiation of TH17 cell (Li et al., 2013). $\beta$-arrestin1 was one member of $\beta$-arrestins, and functioned as multiprotein scaffolds in diverse cellular processes to modulate complex regulating pathways, such as cell proliferation and migration, as well as inflammatory reaction (Talbot et al., 2012; Li et al., 2013). ARRB1 was the phenotype of $\beta$ arrestin1, and our study found that the expression of ARRB1 was associated with liver fibrosis, indicating $\beta$ arrestin1 might also serves as an important biomarker of liver fibrosis. Interestingly, a previous study demonstrated that $\beta$-arrestin1 knockdown reduced the production of TH17, as well as the cytokines that released from $\mathrm{TH} 17(\mathrm{Li}$ et al., 2013), while, our study revealed that the $\mathrm{CCl}_{4}$ induced liver fibrosis increased the level of TH17 and its cytokines, furthermore, the expression of ARRB1 was also increased in the fibrosis liver, their positive correlation was accordance with the previous study. Taken these observations together, we found that ABBR1 could selectively acted as the associated factor of TH17 and liver fibrosis.

It has been widely reported that the dysregulated miRNAs could result in multiple diseases, previous studies have indicated that miRNAs control cellular processes, such as cell proliferation, oxidative stress, inflammatory reaction, as well as tumor growth (Cai et al., 2016; Baker et al., 2016; Hill et al., 2015). The analysis of miRNA on liver diseases have been documented several years before, the down-regulations of miR-150 and miR-194 were demonstrated to lead to severe liver fibrosis (Venugopal et al., 2010), while the over-expression of miR-494 acted as an oncogenicmiRNA that regulated liver tumorgensis(Lim et al., 2014). These findings indicated the decisive role of miRNAs on liver function. In this study, miR-29a and 
miR-652 were the two main biomarkers in liver fibrosis, the expression of both miR-29a and miR-652 were remarkably decreased in model mice with liver fibrosis rather than the control mice. Moreover, online database prediction and luciferase reporter assay proved that ARRB1 was the target gene of both miR-29a and miR-652, and they regulated the onset and progression of liver fibrosis cooperatively.

To better understand the role of miR-29a and miR-652 in liver fibrosis, CD4+ T cells isolated from spleen of normal mice was transfected with miR-29a mimic/miR-652 mimic, which prominently decreased the expression of ARRB1, as well as the level of Th17, IL-17A, IL-22. While, the decreased expression of ARRB1 was reversed by the overexpression of ARRB1. Additionally, in vivo experiments also revealed that overexpression of miR-29a/miR-652 significantly decreased the ALT, AST, Th17, as well as ARRB1, indicating the protective effects of overexpressed miR-29a/miR-652 on liver fibrosis.

In summary, we found that miR-29a and miR-652 were low-expressed while ARRB1 was high expressed in the CD4+ T cells of liver fibrosis mice. ARRB1 mediated by miR-29a and miR-652 was involved in the pathogenesis in liver fibrosis. MiR-29a, miR-652 and ARRB1 were all the main biomarkers in the further clinical diagnosis and therapy of liver fibrosis.

Acknowledgments. This study was supported by Youth Development Projects of Army Medical Technology (15QNP021), Medical and Health Research Projects of Army (15MS063) and Science and Technology Project of Nanjing (201605061).

\section{References}

Baker, J.R., Vuppusetty, C., Colley, T., Papaioannou, A.I., Fenwick, P., Donnelly, L., Ito, K., and Barnes, P.J. 2016. Oxidative stress dependent microRNA-34a activation via PI3Kalpha reduces the expression of sirtuin-1 and sirtuin-6 in epithelial cells. Sci. Rep., 6: 35871.

Bruce, J.P. and Liu, F.F. 2014. MicroRNAs in nasopharyngeal carcinoma. Chin. J. Cancer, 33: 539-544.

Cai, Y.J., Zheng, X.F., Lu, C.H., Jiang, Q., Liu, Q., and Xin, Y.H. 2016. Effect of FUT3 gene silencing with miRNA on proliferation, invasion and migration abilities of human KATO-III gastric cancer cell line. Cell. Mol. Biol. (Noisy-le-grand), 62: 15-20.

Gu, L., Deng, W.S., Sun, X.F., Zhou, H., and Xu, Q. 2016. Rapamycin ameliorates CCl4-induced liver fibrosis in mice through reciprocal regulation of the Th17/Treg cell balance. Mol. Med. Rep., 14: 1153-1161.

Guo, L., Zhao, Y., Yang, S., Zhang, H., and Chen, F. 2014. Integrative analysis of miRNA-mRNA and miRNA-miRNA interactions. Biomed. Res. Int., 2014: 907420

Hernandez-Gea, V. and Friedman, S.L. 2011. Pathogenesis of liver fibrosis. Annu. Rev. Pathol., 6: 425-456.

Hill, J.M., Clement, C., Zhao, Y., and Lukiw, W.J. 2015. Induction of the pro-inflammatory NF-kB-sensitive miRNA-146a by human neurotrophic viruses. Front. Microbiol., 6: 43.

Justinger, C., Kouladouros, K., Gartner, D., Tatsch, K., Reimer, P., Rudiger, T., Binnenhei, M., Bentz, M., and Schon, M.R. 2015. Liver resection after selective internal radiotherapy (SIRT): Proof of concept, initial survival, and safety. J. Surg. Oncol., 112: 436-442.

Kochanek, K.D., Arias, E., and Bastian, B.A. 2016. The Effect of Changes in Selected Age-specific Causes of Death on Non-Hispanic White Life
Expectancy Between 2000 and 2014. NCHS Data Brief, 1-8.

Li, H., Hu, Y., Li, N., and Zhou, Y. 2012a. Liver fibrosis and five year survival of hepatocellular cancer cases undergoing transcatheter arterial chemo embolization using small doses. Asian Pac. J. Cancer Prev., 13: 1589-1593.

Li, J., Qiu, S.J., She, W.M., Wang, F.P., Gao, H., Li, L., Tu, C.T., Wang, J.Y., Shen, X.Z., and Jiang, W. 2012b. Significance of the balance between regulatory $\mathrm{T}$ (Treg) and T helper 17 (Th17) cells during hepatitis B virus related liver fibrosis. PLoS One, 7: e39307.

Li, J., Wei, B., Guo, A., Liu, C., Huang, S., Du, F., Fan, W., Bao, C., and Pei, G. 2013. Deficiency of beta-arrestin1 ameliorates collagen-induced arthritis with impaired TH17 cell differentiation. Proc. Natl. Acad. Sci. USA, 110: 7395-7400.

Lim, L., Balakrishnan, A., Huskey, N., Jones, K.D., Jodari, M., Ng, R., Song, G., Riordan, J., Anderton, B., Cheung, S.T., Willenbring, H., Dupuy, A., Chen, X., Brown, D., Chang, A.N., and Goga, A. 2014. MicroRNA-494 within an oncogenic microRNA megacluster regulates $\mathrm{G} 1 / \mathrm{S}$ transition in liver tumorigenesis through suppression of mutated in colorectal cancer. Hepatology, 59: 202-215.

Moretto, M., Kupski, C., Da Silva, V.D., Padoin, A.V., and Mottin, C.C. 2012. Effect of bariatric surgery on liver fibrosis. Obes. Surg., 22: 1044-1049.

Park, M., Kim, Y.H., Woo, S.Y., Lee, H.J., Yu, Y., Kim, H.S., Park, Y.S., Jo, I., Park, J.W., Jung, S.C., Lee, H., Jeong, B., and Ryu, K.H. 2015. Tonsil-derived mesenchymal stem cells ameliorate CCl4-induced liver fibrosis in mice via autophagy activation. Sci. Rep., 5: 8616 .

Pellicoro, A., Ramachandran, P., Iredale, J.P., and Fallowfield, J.A. 2014. Liver fibrosis and repair: immune regulation of wound healing in a solid organ. Nat. Rev. Immunol., 14: 181-194.

Roderburg, C., Urban, G.W., Bettermann, K., Vucur, M., Zimmermann, H., Schmidt, S., Janssen, J., Koppe, C., Knolle, P., Castoldi, M., Tacke, F., Trautwein, C., and Luedde, T. 2011. Micro-RNA profiling reveals a role for miR-29 in human and murine liver fibrosis. Hepatology, 53: 209-218.

Roderburg, C., Mollnow, T., Bongaerts, B., Elfimova, N., Vargas Cardenas, D., Berger, K., Zimmermann, H., Koch, A., Vucur, M., Luedde, M., Hellerbrand, C., Odenthal, M., Trautwein, C., Tacke, F., and Luedde, T. 2012. Micro-RNA profiling in human serum reveals compartment-specific roles of miR-571 and miR-652 in liver cirrhosis. PLoS One, 7: e32999.

Roderburg, C. and Luedde, T. 2014. Circulating microRNAs as markers of liver inflammation, fibrosis and cancer. J. Hepatol., 61: 1434-1437.

Schenten, D., Nish, S.A., Yu, S., Yan, X., Lee, H.K., Brodsky, I., Pasman, L., Yordy, B., Wunderlich, F.T., Bruning, J.C., Zhao, H., and Medzhitov, R. 2014. Signaling through the adaptor molecule MyD88 in CD4+ T cells is required to overcome suppression by regulatory T cells. Immunity, 40: 78-90.

Shigematsu, K., Nakano, H., Watanabe, Y., Sekimoto, T., Shimizu, K., Nishizawa, A., Makino, M., Okumura, A., Bando, K., and Kitagawa, Y. 2013. Characteristics, risk factors and mortality of stroke patients in Kyoto, Japan. BMJ Open, 3.

Smith, J.S. and Rajagopal, S. 2016. The beta-Arrestins: Multifunctional Regulators of G Protein-coupled Receptors. J. Biol. Chem., 291: 89698977.

Sun, W.Y., Song, Y., Hu, S.S., Wang, Q.T., Wu, H.X., Chen, J.Y., and Wei, W. 2013. Depletion of beta-arrestin2 in hepatic stellate cells reduces cell proliferation via ERK pathway. J. Cell. Biochem., 114: 1153-1162.

Sun, X.F., Gu, L., Deng, W.S., and Xu, Q. 2014. Impaired balance of T helper $17 / \mathrm{T}$ regulatory cells in carbon tetrachloride-induced liver fibrosis in mice. World J. Gastroenterol., 20: 2062-2070.

Talbot, J., Joly, E., Prentki, M., and Buteau, J. 2012. beta-Arrestin1mediated recruitment of $\mathrm{c}$-Src underlies the proliferative action of glucagon-like peptide-1 in pancreatic beta INS832/13 cells. Mol. Cell. 
Endocrinol., 364: 65-70.

Van De Bunt, M., Gaulton, K.J., Parts, L., Moran, I., Johnson, P.R., Lindgren, C.M., Ferrer, J., Gloyn, A.L., and Mccarthy, M.I. 2013. The miRNA profile of human pancreatic islets and beta-cells and relationship to type 2 diabetes pathogenesis. PLoS One, 8: e55272.

Venugopal, S.K., Jiang, J., Kim, T.H., Li, Y., Wang, S.S., Torok, N.J., Wu, J., and Zern, M.A. 2010. Liver fibrosis causes downregulation of miRNA-150 and miRNA-194 in hepatic stellate cells, and their overexpression causes decreased stellate cell activation. Am. J. Physiol. Gastrointest. Liver Physiol., 298: G101-106.

Vishnoi, A. and Rani, S. 2017. MiRNA Biogenesis and Regulation of Diseases: An Overview. Methods Mol. Biol., 1509: 1-10.

Wu, S.G., Huang, Y.J., Bao, B., Wu, L.M., Dong, J., Liu, X.H., Li, Z.H., Wang, X.Y., Wang, L., Chen, B.J., and CHEN, W. 2016. miR-508-5p acts as an anti-oncogene by targeting MESDC1 in hepatocellular carcinoma. Neoplasma, 64.
Xu, H., Hu, Y.W., Zhao, J.Y., Hu, X.M., Li, S.F., Wang, Y.C., Gao, J.J., Sha, Y.H., Kang, C.M., Lin, L., Huang, C., Zhao, J.J., Zheng, L., and Wang, Q. 2015. MicroRNA-195-5p acts as an anti-oncogene by targeting PHF 19 in hepatocellular carcinoma. Oncol. Rep., 34: 175-182.

Yan, S., Yim, L.Y., Lu, L., Lau, C.S., and Chan, V.S. 2014. MicroRNA Regulation in Systemic Lupus Erythematosus Pathogenesis. Immune Netw., 14, 138-148.

Zhang, S., Liang, R., Luo, W., Liu, C., Wu, X., Gao, Y., Hao, J., Cao, G., Chen, X., Wei, J., Xia, S., Li, Z., Wen, T., Wu, Y., Zhou, X., Wang, P., Zhao, L., Wu, Z., Xiong, S., Gao, X., Gao, X., Chen, Y., Ge, Q., Tian, Z., and Yin, Z. 2013. High susceptibility to liver injury in IL-27 p28 conditional knockout mice involves intrinsic interferon-gamma dysregulation of CD4+ T cells. Hepatology, 57: 1620-1631.

(Received for publication, February 23, 2017, accepted, May 13, 2017 and published online, August 2, 2017) 